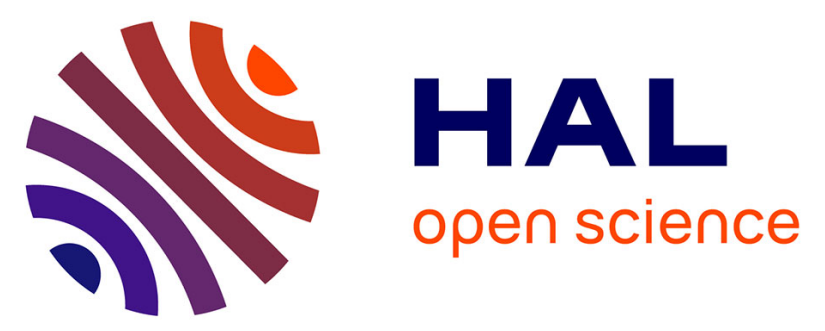

\title{
Passive breakups of isolated drops and one-dimensional assemblies of drops in microfluidic geometries: experiments and models.
}

Louis Salkin, Alexandre Schmit, Laurent Courbin, Pascal Panizza

\section{- To cite this version:}

Louis Salkin, Alexandre Schmit, Laurent Courbin, Pascal Panizza. Passive breakups of isolated drops and one-dimensional assemblies of drops in microfluidic geometries: experiments and models.. Lab on a Chip, 2013, 13 (15), pp.3022-32. 10.1039/c3lc00040k . hal-00844517

\section{HAL Id: hal-00844517 https://hal.science/hal-00844517}

Submitted on 6 Dec 2013

HAL is a multi-disciplinary open access archive for the deposit and dissemination of scientific research documents, whether they are published or not. The documents may come from teaching and research institutions in France or abroad, or from public or private research centers.
L'archive ouverte pluridisciplinaire HAL, est destinée au dépôt et à la diffusion de documents scientifiques de niveau recherche, publiés ou non, émanant des établissements d'enseignement et de recherche français ou étrangers, des laboratoires publics ou privés. 


\title{
Passive breakups of isolated drops and one-dimensional assemblies of drops in microfluidic geometries: experiments and models
}

\author{
Louis Salkin, Alexandre Schmit, Laurent Courbin, ${ }^{*}$ and Pascal Panizza ${ }^{\dagger}$ \\ IPR, UMR CNRS 6251, Campus Beaulieu, Université Rennes 1, 35042 Rennes, France
}

(Dated: October 15, 2013)

\begin{abstract}
Using two different geometries, rectangular obstacles and asymmetric loops, we investigate the breakup dynamics of deformable objects such as drops and bubbles confined in microfluidic devices. We thoroughly study two distinct flow configurations that depend on whether object-to-object hydrodynamic interactions are allowed. When such interactions are introduced, we find that the volumes of the daughter objects created after breakup solely depend on the geometrical features of the devices and is not affected by the hydrodynamic and physicochemical variables; these results are in sharp contrast with those obtained for non-interacting objects. For both configurations, we provide simple phenomenological models that well capture experimental findings and predict the evolution of the volumes of the daughter objects with the controlling dimensionless quantities that are identified. We introduce a mean-field approximation which permits to account for the interactions between objects during breakup and we discuss its conditions of validity.
\end{abstract}

PACS numbers: 47.60.Dx 47.55.D- 47.55.df

\section{INTRODUCTION}

The use of liquid droplets as micron-sized analogs of test tubes, i.e. as microreactors, is a promising route towards the design of high-throughput microfluidics applications [1-4] that aim at analyzing faster and at a lower cost than conventional techniques. This approach known as digital microfluidics $[2,3]$ relies on the ability to generate periodic trains of monodisperse droplets, to handle the drops individually, and to perform and combine basic operations on these deformable objets, e.g. fragmentation, dilution, mixing, and sorting. In contrast to the classical top-down emulsification techniques for which large amounts of droplets are handled at the same time, this technology permits to manipulate individual drops with a precise control of their size and inner composition. This original bottom-up approach makes microfluidics a powerful tool for material and emulsion sciences [5] to create new materials, e.g. double emulsions with inner droplets of different compositions [6], multiple emulsions $[7,8]$, Janus $[9,10]$ or core-shells $[11,12]$ particles.

The development of applications requires to understand the physics behind the basic operations mentioned above. In particular, many recent works have focused on the breakup of drops in confined geometries [13-25]. Indeed, digital microfluidics is a robust tool for highthroughput biological assays [26], droplet splitting being employed for instance for on-chip Polymerase Chain Reaction (PCR) [27] and the screening of compound libraries [28]. An external controlling parameter, e.g. an electric field [24], can be used to break droplets in microfluidic devices. However, most works rely on passive (geometry-based) methods that do not require to use an

\footnotetext{
* laurent.courbin@univ-rennes1.fr

† pascal.panizza@univ-rennes1.fr
}

external field. The pioneering work on the topic [13] describes the two main geometry-mediated methods that can be employed. A train of droplets flowing in a channel can either reach a junction [13-21], that is, the inlet node of a loop having generally two arms of different lengths, or be directed towards a micro-obstacle [13, 22, 23]. All these works show that breakup only occurs when the capillary number exceeds a minimum value. Above this value, all the droplets of a train successively break into two daughter droplets that flow in each of the gaps of an obstacle or in the two arms of a loop depending on the studied geometry. When drops fragment against obstacles, experiments show that the size of the daughter droplets depends on the governing parameters at play: the volume and velocity of the drops, the viscosities of the two immiscible fluids, the surface tension, and the geometrical parameters [22]. By contrast, when loops are used, the experimental size ratio of the produced droplets solely depends on the geometrical features of the device [13]; using successive loops, this method helps to increase the production rate of monodisperse emulsions [29]. The investigations on geometry-mediated breakups almost exclusively describe the various hydrodynamic regimes that can be observed and experimental conditions required to witness drop fragmentation [1325]. On the other hand, the evolution of the size of the daughter objects created upon breakup with the governing parameters has been well studied experimentally but is still poorly understood. Modeling this size is indeed a challenging task because of the large number of parameters potentially at play and possible object-to-object interactions which induce time-delayed feedback [30-34]; because of such feedbacks, the breakup of an object may be influenced by the behavior of the preceding objects.

Here, we aim to address this question working both with isolated and interacting drops and bubbles using the two different methods, obstacles and asymmetric loops. We begin with a brief presentation of results recently 
obtained in the case of isolated drops flowing against obstacles [23]. We describe the basic elements necessary to develop a model that captures most of experimental findings, accounts for the four hydrodynamic regimes observed experimentally, and describes the transitions between these regimes [23]. We then show that this model can also predict the volumes of the daughter drops or bubbles produced when breakup occurs. Afterwards, we perform systematic experiments with rectangular obstacles and asymmetric loops that are very long compared to the distance between objects. In both cases, we identify the physical parameters controlling the volume of the daughter objects. Our experiments show that the set of governing parameters depends on whether the objects are isolated or interacting. Building on previous works on the dynamics of droplet traffic at a junction [35, 36], we introduce a "mean-field" approximation that allows us to treat analytically the problem when object-to-object interactions are present. The predictions obtained for the volume of the created drops after breakup concur very well with experiments. This model also explains a conjectured result reported in [13].

\section{SET-UP}

We carry out experiments with microfluidic devices, fabricated in poly-dimethylsiloxane (PDMS) using soft lithography techniques [37]. The microchannels have a rectangular cross-section $h w$ with an height $h=45 \mu \mathrm{m}$ and a width $w=130 \mu \mathrm{m}$ (see Fig. 1). A periodic train (frequency $f$ ) of monodisperse droplets (or bubbles) is generated using a flow focusing method [38]. In the case of drops, we use syringe pumps (PHD 2000, Harvard Apparatus) to inject the dispersed (an aqueous solution) and continuous (an oil) phases at controlled flow rates which are adjusted independently until a steady flow of monodisperse drops with a desired size $L_{d}=150-900 \mu \mathrm{m}$ is obtained. Typical values of the flow rates for the dispersed and continuous phases are $q_{w}=5-200 \mu \mathrm{l} / \mathrm{hr}$ and $q_{o}^{f}=5-500 \mu \mathrm{l} / \mathrm{hr}$, respectively [Fig. 1(a)]. We employ a similar procedure to generate monodisperse trains of bubbles, besides the injection of the dispersed phase (nitrogen gas) that is performed at constant pressure, 50-700 mbar, using a microfluidic flow control system (MFCS-FLEX, Fluigent). The train is directed towards a linear obstacle of length $L=200-800 \mu \mathrm{m}$ and a width $30 \mu \mathrm{m}$ that is parallel to the walls of the channel but is off-centered so that objects may flow in two gaps (1) and (2) having different widths $w_{1}$ and $w_{2}<w_{1}$ respectively, with $W=w_{2} / w_{1}=0.4-0.9$ characterizing the asymmetry of the obstacle [see Fig. 1(b)]. We only consider situations for which $L_{d}$ is larger than $w$ so that the drops (or bubbles) adopt a shape that resembles a pancake; in what follows, we refer to these large objects as "slugs". An additional injection of the continuous phase at a constant flow rate $q_{o}^{d}=0-1000 \mu \mathrm{l} / \mathrm{hr}$ performed far upstream from the obstacle permits to vary the velocity (a)

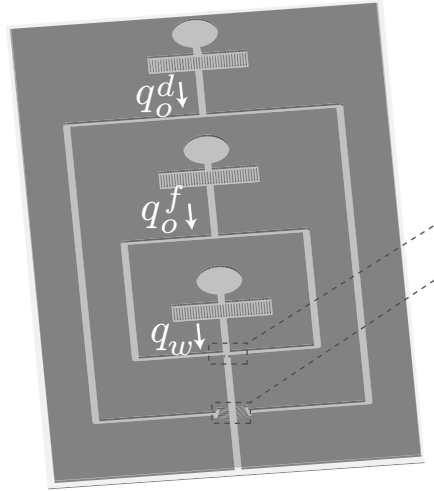

droplet trains production

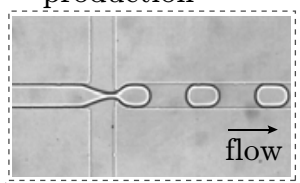

dilution module

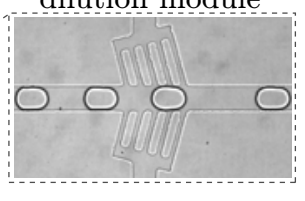

(b)
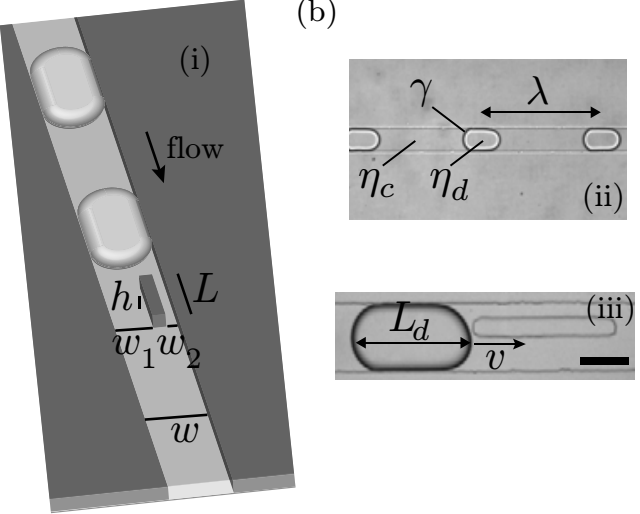

FIG. 1. (a) Schematic of the microfluidic device upstream of the obstacle and photographs showing the two main modules used to create periodic trains of monodisperse slugs. Defined are the oil flow rates in the production and dilution modules, $q_{o}^{f}$ and $q_{o}^{d}$, and the flow rate of the slugs phase $q_{w}$. (b) (i) Schematic of the flow-geometry nearby the obstacle defining the geometrical parameters $h, L, w, w_{1}$, and $w_{2}$. (ii) and (iii) Photographs defining the hydrodynamics and physicochemical variables $\gamma, \eta_{c}, \eta_{d}, \lambda, v$, and $L_{d}$. Scale bar: $100 \mu \mathrm{m}$.

of the slugs $v$ and the distance between slugs $\lambda$ while maintaining their size, thus their volume $\Omega=q_{w} / f$, unchanged [32]. In our experiments, the Reynolds and the capillary numbers are small and span the ranges $10^{-3}-10^{-1}$ and $10^{-3}-10^{-2}$, respectively. Images of the flow are recorded close to the obstacle with a high-speed camera (Phantom V7, Vision Research) working at typical frame rates of 500-5000 frames/s. The motion of the slugs as well as their size are obtained from image analysis using a custom-written software developed with MATLAB. The liquid-liquid systems are water-glucose mixtures (viscosity $\eta_{d}=1$ to $7 \mathrm{mPa} . \mathrm{s}$ ) dispersed in either hexadecane (viscosity $\eta_{c}=3 \mathrm{mPa} . \mathrm{s}$ ) or a viscous silicone oil $\left(\eta_{c}=50 \mathrm{mPa} . \mathrm{s}\right)$. The dispersed phase contains $15 \mathrm{~g} / \mathrm{L}$ of a surfactant (Sodium Dodecyl Sulfate, Sigma). The gas-liquid system is a nitrogen gas in hexadecane. For each system, we measure the surface tension $\gamma$ between dispersed and continuous phases with pendant drop (or bubble) tensiometry. 


\section{FRAGMENTATION OF ISOLATED DROPS AND BUBBLES}

\section{A. Experiments: hydrodynamic regimes}

We begin by studying the fragmentation of isolated drops and bubbles against a rectangular obstacle. This situation is obtained when the slugs are sufficiently far apart so that whenever the front edge of a slug meets the obstacle, the two gaps (1) and (2) are only filled with the continuous phase. In such a configuration, the fragmentation of a slug does not affect the breakup dynamics of the following slug and a drop or bubble can therefore be considered as non-interacting, i.e. isolated. This situation is obtained experimentally when $\lambda \gg L$.

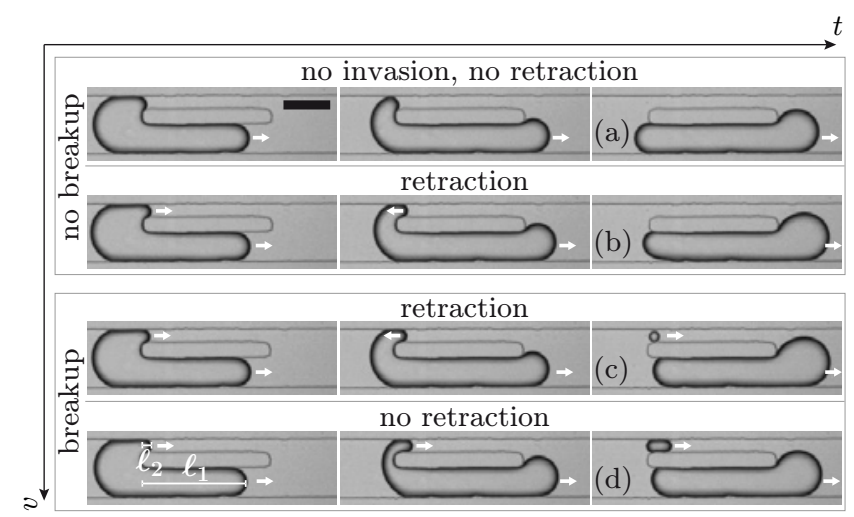

FIG. 2. (a)-(d) Four different hydrodynamic regimes are observed when isolated slugs of the same size flow against an obstacle at different speeds; adapted from [23]. The position of an interface in the $(i)$-th gap, $i=1$ or 2 , is defined by $\ell_{i}(t)$; the slug meets the obstacle at a time $t=0$. Scale bar: $100 \mu \mathrm{m}$.

For $\eta_{d}>\eta_{c}$ and a fixed value of $L_{d}$, we observe the behavior of isolated slugs flowing against the same rectangular obstacle at different velocities $v$. We obtain four different hydrodynamic regimes (Fig. 2). A slug may or may not break against the obstacle, these regimes occurring with [Figs. 2(b)-(c)] or without [Figs. 2(a)-(d)] the retraction of two-fluid interface in the narrow gap (2). More specifically, for small enough speeds [see Figs. 2(a)(b)], a slug does not not break and flows through the large gap (1). When the speed is sufficiently large, breakup occurs with or without the retraction of an interface in the gap (2) [see Figs. 2(c)-(d)]. In a recent study [23], we have systematically studied the occurrence of these regimes as a function the governing parameters shown in Fig. 1(b). This earlier work reports a theoretical framework which well describes experimental findings in terms of the seven dimensionless quantities controlling the dynamics. This model also provides diagrams mapping the four hydrodynamic regimes found experimentally. In the next section, we give the basic elements necessary to derive this model.

\section{B. Modeling the flow}

\section{Transport of slugs}

Building on previous models describing the transport of slugs in microfluidic conducts at low Reynolds and capillary numbers $[20,22,23]$, we assume that $v$ varies as $v=\frac{q}{h w}$ with $q=q_{w}+q_{o}^{f}+q_{o}^{d}$ the total flow rate, and that Darcy's laws describe the flows of both the continuous phase and the slugs phase with an effective viscosity $\eta_{s}\left(h, w, \eta_{d}, \eta_{c}\right)$ for the slugs. This phenomenological effective viscosity accounts for additional viscous dissipation inside the slug, in the thin films of continuous phase between the slug and the channel walls, floor, and ceiling, and in the corners of the rectangular geometry. The pressure drop $\Delta p$ over a portion $\ell$ of a slug then reads $\Delta p=\frac{\eta_{s} \ell}{h^{3} w} f\left(\frac{w}{h}\right) q$, where $f\left(\frac{w}{h}\right) \approx 12\left(1-0.63\left(\frac{w}{h}\right)^{-1}\right)^{-1}$ in the situation $(h<w)$ considered here [39]. Due to the curved two-fluid interfaces, there are also pressure drops across the front and rear edges of the slug, which we write approximately as $\Delta p_{\text {curv }}=\epsilon \frac{2 \gamma}{w}\left(1+\frac{w}{h}\right)$, with $\epsilon=1$ or -1 depending on whether the front or the rear edge of the slug is considered. Because of its spatial periodicity, we model the flow over a length $\lambda$, within two distinct regions of lengths $L_{d}$ and $\lambda-L_{d}$ corresponding respectively to the slug and the space between slugs (see Fig. 3). In the situation depicted in Fig. 3, the contribution to the total pressure drop of the curved rear and front slug edges cancel out [40] so that the pressure drop over $\lambda$ is $\Delta p_{\lambda}=\frac{\eta_{c} \lambda}{h^{3} w} f\left(\frac{w}{h}\right)\left[1+\Delta \eta \frac{L_{d}}{\lambda}\right] q$, with the viscosity contrast $\Delta \eta=\frac{\eta_{s}-\eta_{c}}{\eta_{c}}$.

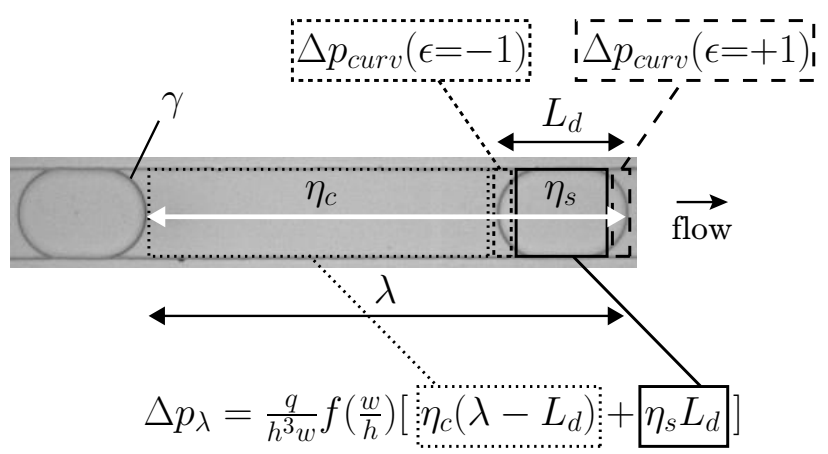

FIG. 3. Photograph of the flow describing the modeling of the pressure drops in our study. Slugs of size $L_{d}$ separated by a distance $\lambda$ flow in a rectangular channel of width $w$ and height $h$. The pressure drop over the length indicated by the white arrow is the sum of $\Delta p_{\lambda}$ corresponding to the flow of the continuous phase and slugs, and the additional pressure drop across the front and rear edges of the slug due to the curved two-fluid interfaces $\Delta p_{\text {curv }}=\epsilon \frac{2 \gamma}{w}\left(1+\frac{w}{h}\right) ; \epsilon=1$ (front edge)or $\epsilon=-1$ (rear edge). This model assumes that the continuous phase and slugs flow at the same speed.

It is worthwhile mentioning that in the case of a long channel of length $\ell \gg \lambda$ the pressure drop is $\Delta p \simeq \frac{\ell}{\lambda} \Delta P_{\lambda}$ 
so that the hydrodynamic resistance of the channel reads $\frac{\Delta p}{q} \simeq \frac{\eta_{c} \ell}{h^{3} w} f\left(\frac{w}{h}\right)\left[1+\Delta \eta \frac{L_{d}}{\lambda}\right] \simeq \frac{\eta_{c}}{h^{3} w} f\left(\frac{w}{h}\right)\left(\ell+n L_{r}\right)$ where $n \simeq \frac{\ell}{\lambda}$ is the number of slugs in the channel and $L_{r}=L_{d} \Delta \eta$ has the dimension of a length. This phenomenological model neglecting the dependance of the hydrodynamic resistance on the recirculation around the caps of the slugs, predicts that the hydrodynamic resistance of a channel filled with slugs varies linearly with the number of slugs it contains, with $L_{r}$ an excess length added by each slug to the channel in terms of hydrodynamic resistance. The existence of such a resistive length is also predicted in the case of the transport of spherical drops in microfluidic conduct at low capillary and Reynolds numbers using a slightly different phenomenological approach [41] validated by experiments for both spherical drops and slugs [32, 35, 41-43]. Interestingly, our simple model for the hydrodynamic resistance of a microchannel filled with slugs also predicts that the effective length $L_{r}=L_{d} \Delta \eta$ can be negative when $\Delta \eta<0$. This prediction could inspire further experimental investigations of droplet traffic for fluid-fluid systems having negative viscosity contrasts.

\section{Fragmentation against a rectangular obstacle}

Here, we briefly present the model reported in [23] that allows for a rationalization of the experiments discussed in the section III A. We will then show that this model also predicts the volumes of the daughter drops or bubbles that are created when breakup occurs.

Our model is based on the basic physical arguments discussed in section III B 1. The dynamics start at a time $t=0$ when an isolated slug meets the obstacle. Since our experiments are carried out at constant flow rates, a twofluid interface always enters the large gap (1) and begins to move forward at a speed $v_{1}(t)=d \ell_{1} / d t$ up to a distance $\ell_{1}=X_{1} L$ [see Fig. 2(d) defining $\ell_{1}$ ]. Similarly, in regimes for which a two-fluid interface propagates in the narrow gap, the velocity and location of this interface in gap (2) are $v_{2}(t)=d \ell_{2} / d t$ and $\ell_{2}=X_{2} L$, respectively [Fig. 2(d) defines $\left.\ell_{2}\right]$. In our model, the pressure drop $\Delta p_{\text {curv }}$ accounts for the presence of curved interfaces. However, this simple model is derived considering flat interfaces. Our experiments show that the velocity of the slug $v$ remains constant after collision until the rear edge of the slug reaches the obstacle. Therefore, since we consider slugs having flat interfaces, we assume that this event occurs at a time $t_{f}=\frac{L_{d}-c w}{v} ; c$ is a free parameter $O(1)$ that depends on the dimensionless parameters of the crosssection of the channel, i.e. $\frac{w}{h}, \frac{w_{2}}{h}$, and $\frac{w_{2}}{W h}$. We next work with the dimensionless time $T=\frac{t}{t_{f}}$.

Break-up occurs provided that a two-fluid interface has invaded gap (2) and has not completely withdrawn from this gap at $T=1$. The dynamics of the two-fluid interfaces in both gaps are controlled by a set of two coupled first-order ordinary differential equations [23]. The first equation is given by the conservation of the total flow rate:

$$
\frac{d X_{1}}{d T}+W \frac{d X_{2}}{d T}=\frac{\left(L_{d}-c w\right) w}{L w_{1}}=\alpha .
$$

The equality of pressure drops over both gaps gives the second equation:

$$
\left(1+\Delta \eta X_{1}\right) \frac{d X_{1}}{d T}-F W\left(1+\Delta \eta X_{2}\right) \frac{d X_{2}}{d T}=\alpha \frac{\mathcal{C}_{\star}}{\mathcal{C}}
$$

for $X_{1} \leq 1$ and $X_{2} \leq 1$;

$$
(1+\Delta \eta) \frac{d X_{1}}{d T}-F W\left(1+\Delta \eta X_{2}\right) \frac{d X_{2}}{d T}=\alpha \frac{\mathcal{C}_{\star}}{\mathcal{C}} \frac{1+\frac{w_{2}}{h}}{1-W}
$$

for $X_{1}>1$ and $X_{2} \leq 1 . \quad F=f\left(\frac{w_{2}}{h}\right) /\left[W f\left(\frac{w_{2}}{W h}\right)\right]$ and $\mathcal{C}=\frac{\eta_{c} v}{\gamma}$ is the capillary number. Other dimensionless quantities are $\mathcal{C}_{\star}=2 Z \frac{1-W}{W}$ with $Z=\left(f\left(\frac{w_{2}}{W h}\right) h^{-2} w L\right)^{-1}$.

This set of equations is written is terms of the seven dimensionless quantities controlling the breakup dynamics $\left(W, Z, \frac{w}{h}, \frac{w_{2}}{h}, \mathcal{C}, \frac{L_{d}}{L}, \Delta \eta\right)$. The first four quantities are associated with the geometry of the channel whereas $\mathcal{C}$, $\frac{L_{d}}{L}$, and $\Delta \eta$ depend on the hydrodynamic parameters and fluid properties. Not surprisingly, $\mathcal{C}$, which compares the magnitude of viscous and capillary stresses, appears to be a governing quantity as seen in other problems addressing the breakup of drops or bubbles $[13,20,22,44]$. This theoretical framework captures experimental observations and transitions between hydrodynamic regimes discussed in section III A and reported in Salkin et al. [23].

\section{Size distribution after breakup}

A slug breaks whenever it has entered the narrow gap and $X_{2}(\mathrm{~T}=1)>0$. Using this condition for breakup and solving numerically the set of eqns (1) allows us to determine the volume of the daughter slugs $\Omega_{i} \simeq w_{i} h L X_{i}(1)$ produced in gap $(i)$ with $i=1$ or 2 . Varying the size and velocity of a slug, the fluid viscosities, the surface tension, and the geometrical features of the obstacle, we next compare the predicted volumes $\Omega_{i}$ with experimental measurements. Despite the apparent complexity of a problem with a large number of governing parameters [see Fig. 1(b)], in what follows, we show that our simple model based on strong approximations can predict the daughter slugs size distribution using only two free parameters, the effective viscosity $\eta_{s}$ and the numerical constant $c$ introduced because we consider flat interfaces rather than curved. The results presented below from Fig. 4 through Fig. 6 are discussed in terms of $\phi_{2}=\frac{\Omega_{2}}{\Omega}$ the ratio of the volume $\Omega_{2}$ over the volume of the initial slug $\Omega$ studied as a function of $\mathcal{C}$. As shown in these figures, the numerical simulations provide a good qualitative description of our experimental findings as it captures well the variations of the volume fraction $\phi_{2}$ when experimental parameters are varied. In addition, the values taken 
by the free parameters to determine the best fits to the experimental volume of the daughter slugs are consistent with the free parameters determined in our previous work [23] by fitting the analytical expressions of the transitions separating the four hydrodynamic regimes in the $\left(\mathcal{C}, L_{d}\right)$ plane to the experimental data; these regimes are briefly discussed in section III A.

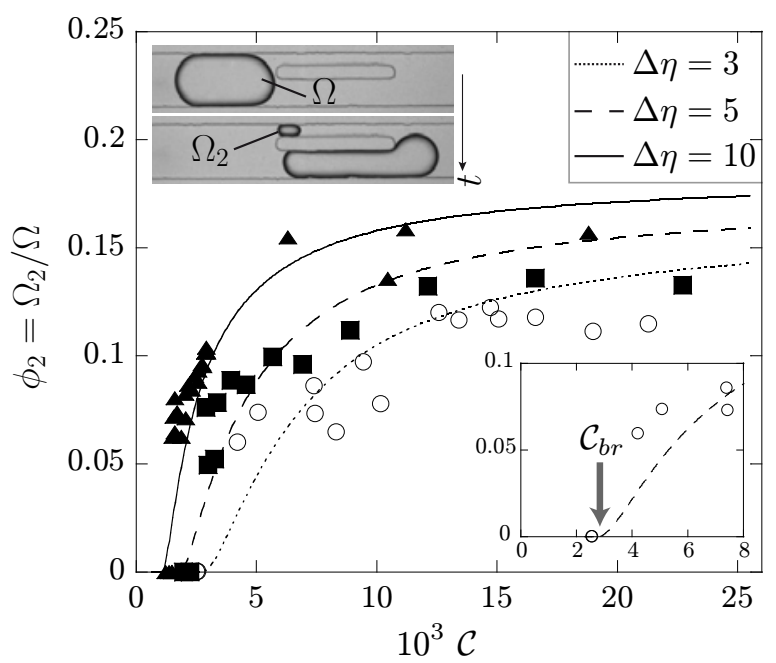

FIG. 4. Evolution as a function of $\mathcal{C}$ of the volume fraction $\phi_{2}$ for different water-glucose mixtures in hexadecane: (o) $\eta_{d}=1 \mathrm{mPa} \mathrm{s},(\boldsymbol{\square}) \eta_{d}=2.7 \mathrm{mPa} \mathrm{s}$, and $(\mathbf{\Delta}) \eta_{d}=7.2 \mathrm{mPa} \mathrm{s}$. The photographs define $\Omega$ and $\Omega_{2}$. Inset: Magnification at low $\phi_{2}$ and $\mathcal{C}$ defining the minimum capillary number for breakup to occur, $\mathcal{C}_{b r}$. The dimensionless quantities are $W=0.4, \frac{w}{h}=2.7$, $\frac{w_{2}}{h}=0.57, Z=2.8 \times 10^{-3}$. The lines are predictions of the model. The two free parameters are $c=0.5$ and (dotted line) $\Delta \eta=3,($ dashed line) $\Delta \eta=5$, and (solid line) $\Delta \eta=10$.

We begin by studying the evolution of $\phi_{2}$ with $\mathcal{C}$ for different liquid-liquid systems, i.e. for different viscosity contrasts. Figure 4 show that above a critical value $\mathcal{C}_{b r}$ of $\mathcal{C}$ a slug breaks into two daughter slugs (see inset of Fig. 4 defining $\mathcal{C}_{b r}$ for a set of data) in accordance with our previous observations [23]. Additionally, when $\mathcal{C}>\mathcal{C}_{b r}, \phi_{2}$ is a monotonically increasing function of $\mathcal{C}$ which indicates that $\Omega_{2}$ becomes larger as $\mathcal{C}$ increases. When breakup occurs, for a given capillary number, $\phi_{2}$ strongly increases with the viscosity contrast. Experiments also show that $\mathcal{C}_{b r}$ becomes smaller when the viscosity contrast is increased. This result indicates that, for $\Delta \eta>0$ and a given continuous phase, the minimum capillary number to break a slug against a rectangular micro-obstacle is smaller for a high-viscosity fluid slug compared to a low-viscosity one. This behavior is surprising as it differs from what is usually observed in bulk for an isolated drop submitted to a flow [44]; it clearly shows that the physics of drop fragmentation is highly sensitive to confinement [45]. The model captures well all experimental observations discussed above (Fig. 4).

We next investigate the effect of the slug size on the fragmentation process. Figure 5 shows the evolution of

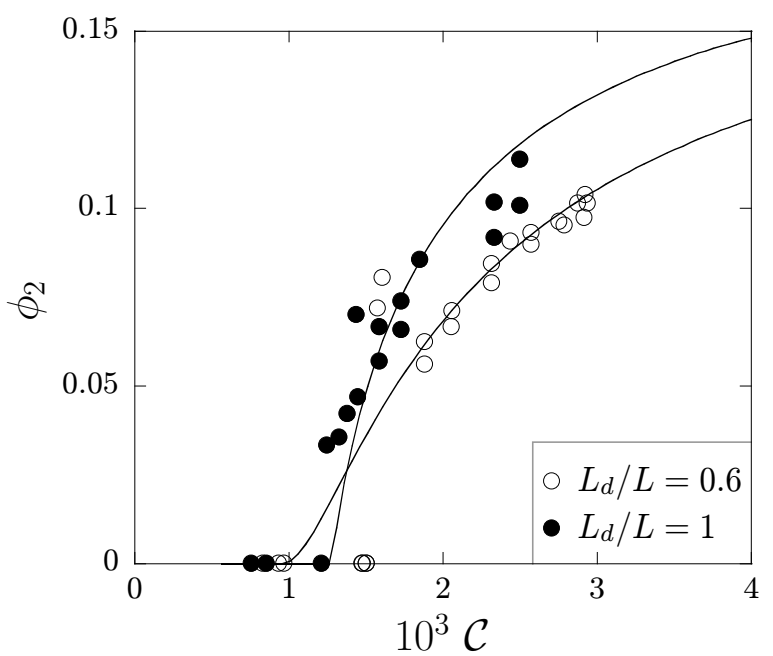

FIG. 5. Variations of $\phi_{2}$ as a function of $\mathcal{C}$ for a water-glucose mixture in hexadecane $\left(\eta_{d}=7.2 \mathrm{mPa} s\right)$ and two values of $L_{d} / L$ respectively smaller and larger than $L_{d}^{c r} / L$ : (०) $L_{d} / L=0.6$ and $(\bullet) L_{d} / L=1$; in these experiments, $L_{d}^{c r} / L=0.74$. The dimensionless quantities are $W=0.4, \frac{w}{h}=2.7, \frac{w_{2}}{h}=0.57$, and $Z=2.8 \times 10^{-3}$. The lines are predictions of the model calculated using the two free parameters $c=0.5$ and $\Delta \eta=10$.

$\phi_{2}$ with $\mathcal{C}$ for two different values of $L_{d} / L$ in the case of a liquid-liquid system whose viscosity contrast is positive $\left(\eta_{c}=3 \mathrm{mPa}\right.$ s and $\eta_{d}=7.2 \mathrm{mPa}$ s). For such viscosity contrasts, we have recently reported the existence of a critical drop size $L_{d}^{c r}$ for which breakup requires a minimum capillary number [23]. When $L_{d}<L_{d}^{c r}$, only breakup regimes occurring without the retraction of a two-fluid interface in the narrow gap are observed. By contrast, depending on the value of $\mathcal{C}$, the fragmentation may or may not occur with such a retraction when $L_{d}>L_{d}^{c r}$; a detailed discussion of these findings is provided in [23]. As shown in Fig. 5, the model describes reasonably well the variations of $\phi_{2}$ when $L_{d} / L$ either smaller or larger than $L_{d}^{c r} / L$. Above $\mathcal{C}_{b r}, \phi_{2}$ monotonically increases with $\mathcal{C}$ and its evolution weakly depends on $L_{d} / L$.

Using different devices, we next study the influence of the obstacle asymmetry $W$ on the evolution of $\phi_{2}$ with $\mathcal{C}$ (Fig. 6); note that the results are discussed in terms of $F=f\left(\frac{w_{2}}{h}\right) /\left[W f\left(\frac{w_{2}}{W h}\right)\right]$ instead of $W$ as $w_{2} / h$ may vary from a device to another. As mentioned previously, experiments can be conducted with bubbles rather than drops. We provide in Fig. 6 a set of results obtained for the gas-liquid system showing that the model well predicts the volumes of both drops and bubbles. In other words, this model can predict the volume of daughter objects for both positive and negative viscosity contrasts. Experiments performed with the liquid-liquid system show that $\mathcal{C}_{b r}$ increases with $F$. The volume fraction $\phi_{2}$ is also sensitive to the obstacle asymmetry: for a given capillary number, $\phi_{2}$ strongly decreases with $F$ (Fig. 6).

To summarize, working within the limit $\lambda \gg L$, we have studied the volume of daughter drops or bubbles obtained 


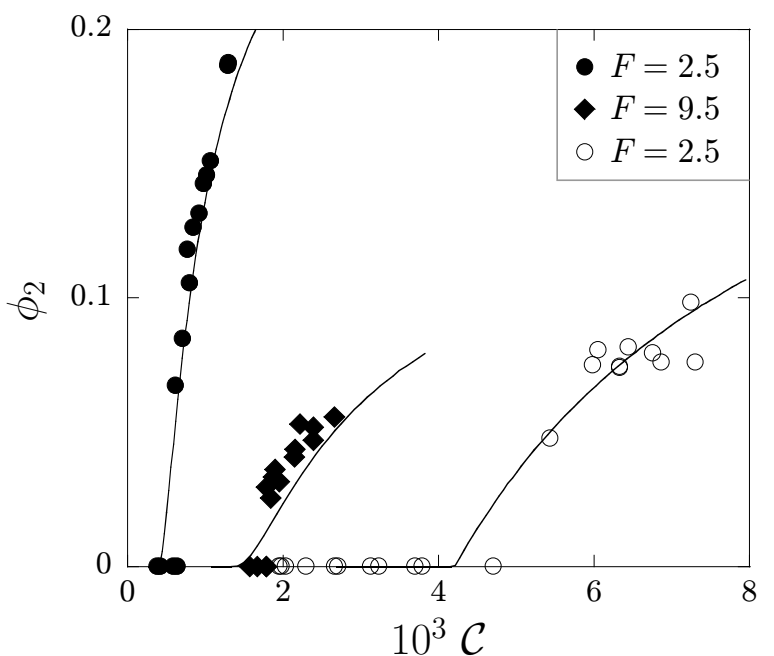

FIG. 6. Evolution of $\phi_{2}$ with $\mathcal{C}$ for different values of $F$. The solid lines correspond to the model for (closed symbols) slugs of the water/glucose mixture in hexadecane $\left(\eta_{d}=7.2 \mathrm{mPa} \mathrm{s}\right)$ and (open symbols) bubbles filled with nitrogen gas in hexadecane. The shape of the symbols stands for different values of $F$ as indicated in the figure. The dimensionless quantities are (o) $W=0.6, \frac{w_{2}}{h}=0.78, Z=3 \times 10^{-3}, L_{d} / L=0.7$, ( ) $W=0.35$, $\frac{w_{2}}{h}=0.54, Z=3.7 \times 10^{-3}, L_{d} / L=0.9$, and $(\bullet) W=0.6, \frac{w_{2}}{h}=0.83$, $Z=3.3 \times 10^{-3}, L_{d} / L=0.9$. For each experiment, $\frac{w}{h}=3.03$. The free parameters are (०) $c=0.49$ and $\Delta \eta=-0.97,(\diamond) c=0.8$ and $\Delta \eta=10$, and $(\bullet) c=0.9$ and $\Delta \eta=10$.

after the breakup of isolated slugs against a rectangular obstacle. Our results show that breakup occurs when the capillary number $\mathcal{C}$ exceeds a minimum value $\mathcal{C}_{b r}$. When $\mathcal{C} \geq \mathcal{C}_{b r}$, daughter slugs are produced in both gaps and the volume fraction in the narrow gap $\phi_{2}$ is an increasing function of $\mathcal{C}$. Although, $\mathcal{C}_{b r}$ is a nontrivial function of the governing parameters of the problem, its variations can be fully captured using the model described in [23]. Experiments show that the volume of the daughter slugs depends on the capillary number, the size of the initial slug, the viscosity contrast, and the asymmetry of the obstacle. Using only two free parameters, the model reported in [23] describes semiquantitatively our experiments. When we compare the viscosity of the dispersed phase $\eta_{d}$ with the values of $\eta_{s}$ determined for the fits to the data in Fig. 4 through Fig. 6, we find that our results concur with the literature. Indeed, when $\Delta \eta>0$, we find that $\eta_{s}>\eta_{c}$ which is consistent with [42]. By contrast, $\eta_{d}<\eta_{s}<\eta_{c}$ when $\Delta \eta<0$ which concurs with other works [43]. In section IV, working within the limit $\lambda \ll L$, we will investigate the breakup in the presence of slugto-slug interactions. When $\lambda \sim L$, we observe the emergence of complex breakup dynamics that includes periodic fragmentation events; these results will be discussed elsewhere [46]. Using a mean-field approach, we determine in the next section the critical value of $\lambda$ below which the slugs can no longer be considered as isolated.

\section{Long obstacles and large capillary numbers: A} mean-field approximation

We consider configurations for which $L_{d} / L$ is small enough to establish a mean-field approximation. In our model considering flat interfaces, this situation corresponds to small enough ratios $L_{d}^{e f f} / L=\left(L_{d}-c w\right) / L$. In this case, a two-fluid interface is still present in the large gap when the rear edge of a slug reaches the obstacle, i.e. $X_{1}(T=1)<1$, and the dynamics of the two-fluid interfaces propagating in both gaps are solely governed by eqn (1a) and eqn (1b). One can then assume that the variations in time of the pressure drops in both gaps associated with the flow of the slug and the presence of curved interfaces remain negligible when compared to the mean values of the pressure drops. This assumption can be mathematically expressed as $|\Delta \eta| X_{i} \ll 1$ and $\mathcal{C} \gg \alpha \mathcal{C}_{\star}$. Hence, eqn (1b) becomes:

$$
\frac{d X_{1}}{d T}-F W \frac{d X_{2}}{d T} \simeq 0
$$

Solving the set of eqns (1a) and (2) one easily finds $\frac{d X_{1}}{d T} \simeq \frac{\alpha F}{(1+F)}$ and $\frac{d X_{2}}{d T} \simeq \frac{\alpha}{W(1+F)}$. Using these relationships, one derives expressions for $\phi_{i}=\Omega_{i} / \Omega$ in the gap $(i)$ with $i=1$ or 2 :

$$
\phi_{2} \simeq \frac{1}{1+F}
$$

and

$$
\phi_{1} \simeq \frac{F}{1+F}
$$

Using these results, the conditions for the validity of our approximation read $\frac{L_{d}^{\text {eff }}}{L}<\frac{w_{1}(1+F)}{w F}, \frac{L_{d}^{\text {eff }}}{L} \ll \frac{w_{1}(1+F)}{w F|\Delta \eta|}$, and $\mathcal{C} \gg \alpha \mathcal{C}_{\star}$. One can also derive an expression for the critical distance between slugs $\lambda_{c}$ above which a slug can be considered as isolated when reaching an obstacle at $T=0$. At this time, when $\lambda \geq \lambda_{c}$ both gaps are only filled with continuous phase, which indicates that the daughter slug created in the narrow gap after the breakup of the previous slug at $T=-\frac{\lambda-L_{d}^{e f f}}{L_{d}^{e f f}}$ has moved by at least a dimensionless distance 1 over the duration $\frac{\lambda-L_{d}^{e f f}}{L_{d}^{e f f}}$. Using $\frac{d X_{2}}{d T} \simeq \frac{\alpha}{W(1+F)}$, when $\lambda=\lambda_{c}$, this simple analysis gives:

$$
\lambda_{c} \simeq L \frac{w_{2}(1+F)}{w}+L_{d}^{e f f} .
$$

For larger $L_{d}^{e f f}$, a two-fluid interface may have left the large gap at $T=1$. This situation is apparently more complex since the dynamics of the two-fluid interfaces are then controlled by the complete set of eqns (1). Nevertheless, when $|\Delta \eta| \ll 1$ and $\mathcal{C} \gg \alpha \mathcal{C}_{\star} \frac{1+\frac{w_{2}}{h}}{1-W}$, eqn (1b) and eqn (1c) both reduce to eqn (2) and a similar analysis gives the same expressions for $\phi_{1}, \phi_{2}$, and $\lambda_{c}$ as determined when $X_{1}(T=1)<1$. 


\section{FRAGMENTATION IN THE PRESENCE OF SLUG-TO-SLUG INTERACTIONS}

\section{A. Experiments}

We now study the breakup dynamics of onedimensional assemblies of slugs for which $\lambda \ll L_{i}$ against obstacles depicted in Fig. 7.
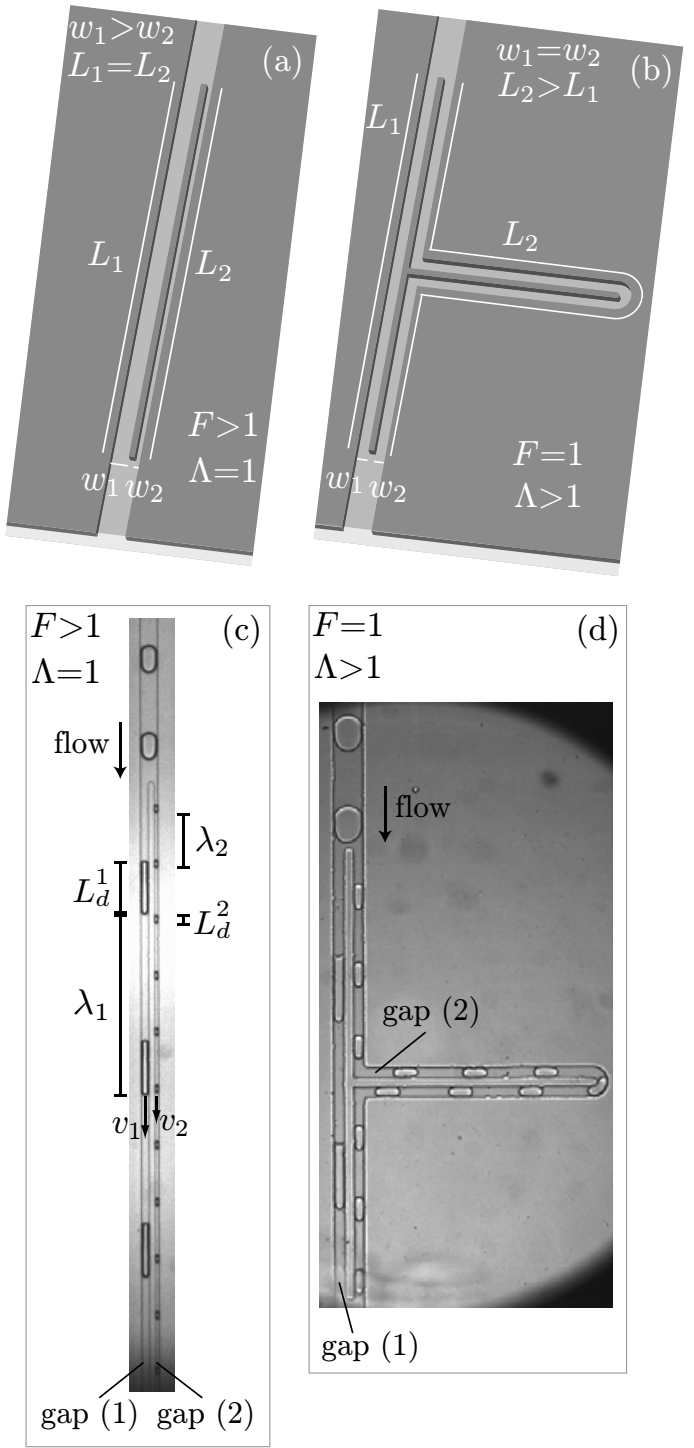

FIG. 7. Sketch of the long rectangular obstacles (a) and asymmetric loops (b). The asymmetry is controlled by $F=f\left(\frac{w_{2}}{h}\right) /\left[W f\left(\frac{w_{2}}{W h}\right)\right]$ and $\Lambda=L_{2} / L_{1}$. Photographs showing typical flows of slugs against long rectangular obstacles (c) and in asymmetric loops (d). Defined are the velocity $v_{i}$ of the slugs produced in the $(i)$-th gap $(i=1$ or 2$)$ after breakup, their size $L_{d}^{i}$, and the distance $\lambda_{i}$ between them.

The two gaps (1) and (2) of the obstacles have lengths $L_{1}$ and $L_{2}$ and widths $w_{1}$ and $w_{2}$ (Fig. 7); $L_{i}=10^{3}-10^{4} \mu \mathrm{m}$ with $i=1$ or 2 . These obstacles are ei- ther long and rectangular [Fig. 7(a)] or can be viewed as long asymmetric loops [Fig. 7(b)]. $\quad F$ and $\Lambda=\frac{L_{2}}{L_{1}}$ characterize the asymmetry: $F>1$ and $\Lambda=1$ for rectangular obstacles whereas $F=1$ and $\Lambda>1$ in the case of loops.

We work within the limits of long obstacles $\left(\lambda \ll L_{i}\right)$ since, for this flow configuration, one may assume that the temporal fluctuations of the total flow rates in each gap of an obstacle can be neglected; this will help to rationalize our results in section IVB. As we wish to study the volumes of daughter slugs after breakup, we work with large enough capillary numbers so that all the slugs of a train break when meeting an obstacle. In this case, experiments show that whenever the front edge of a slug meets the obstacle, two fluid-fluid interfaces invade both gaps, propagating in the gap $(i)$ at a speed $v_{i}$ [see Fig. 7(c)-(d)]. The rear edge of this slug collides with the obstacle at a time $t_{f}$ defined in the section III B 2. At $t_{f}$, the slug breaks into two daughters slugs ( size $L_{d}^{i}=v_{i} t_{f}$ ) that flow in the two gaps (see Fig. 7 defining $L_{d}^{1}$ and $L_{d}^{2}$ ). The time between two successive breakup events being $\tau=\frac{1}{f}=\frac{\lambda}{v}$, the distance between the front edges of two consecutive slugs flowing in gap $(i)$ is $\lambda_{i}=v_{i} \tau$. In what follows, we study the volume fraction $\phi_{2}$ as a function of $\mathcal{C}, L_{d}, \eta_{d}, F$, and $\Lambda$.

We begin by investigating the breakup dynamics in rectangular geometries $(\Lambda=1$ and $F>1)$. For a given fluid-fluid system and size of the slug, our experiments show that $\phi_{2}$ does not depend on $\mathcal{C}$ (Fig. 8). Furthermore, figure 8 shows that $\phi_{2}$ is independent of the viscosity contrast for the range of studied capillary numbers.

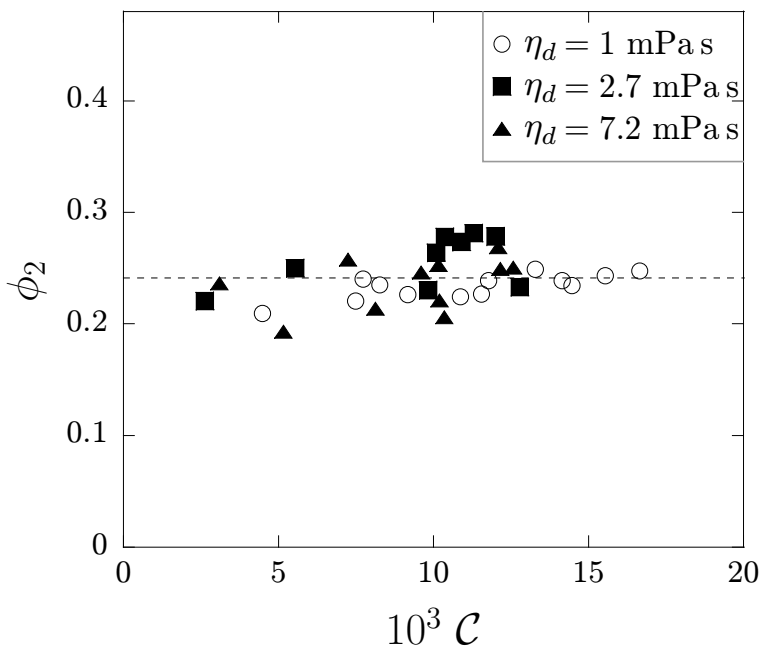

FIG. 8. Evolution of $\phi_{2}$ with $\mathcal{C}$ for a fixed slug size and geometry $\left(L_{d}=230 \pm 30 \mu \mathrm{m}\right.$ and $\left.F=2.5\right)$ and different waterglucose mixtures in hexadecane, i.e. different values of $\eta_{d}$.

For a given geometry and capillary number, we report in Fig. 9 the variations of $\phi_{2}$ with the slug size $L_{d}$. For three different values of $\eta_{d}$, experiments show that $\phi_{2}$ remains constant when $L_{d}$ is varied and roughly equal to the quarter of the volume of the initial slug. 


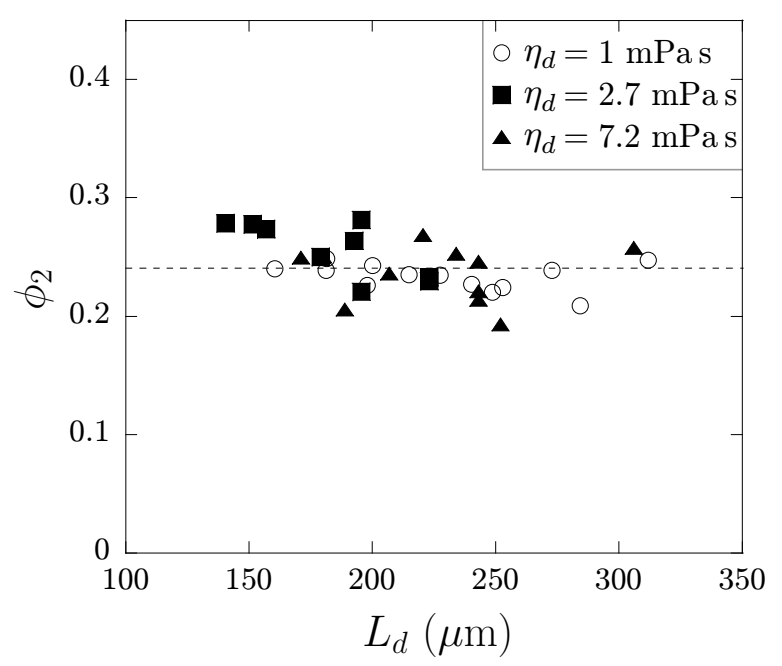

FIG. 9. Variations of $\phi_{2}$ with $L_{d}$ for three different values of $\eta_{d}$. The parameter controlling the asymmetry is $F=2.5$.

By contrast, the response is sensitive to the asymmetry as $\phi_{2}$ roughly decreases from 0.5 to 0.1 when $F$ is varied between 1 and 6 (Fig. 10).

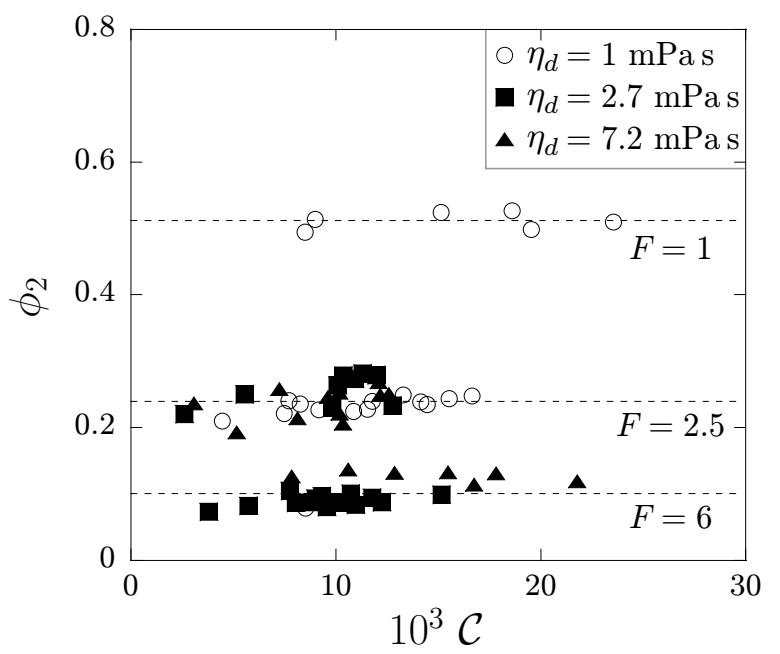

FIG. 10. Evolution of $\phi_{2}$ as a function of $\mathcal{C}$ for different values of $F$ and $\eta_{d}$ as indicated in the figure.

To summarize, the volume fraction $\phi_{i}$ does not depend on hydrodynamic parameters and physicochemical properties and is a decreasing function of the asymmetry of the obstacle. These results contrast with our findings for isolated drops discussed in section III C. In the next section, we will develop a phenomenological model based on a mean-field approximation to rationalize our experiments. We will show that this model concur well with experimental results obtained both for rectangular obstacles and asymmetric loops (Fig. 11 and Fig. 12).

\section{B. Interpretation: a mean-field approach}

We have investigated the flow of periodic trains made of monodisperse slugs for which $\lambda \ll L_{i}$ working with large enough capillary numbers so that all slugs of a train successively break when meeting the obstacle. It is interesting to note that the sole occurrence of breakup regimes without retraction, although not intuitive for loops, could have been expected for long obstacles, since the minimum capillary number to break a slug vary as $1 / L$ for an isolated slug flowing against a rectangular micro-obstacle [23]. Furthermore, when $\lambda \ll L_{i}$, the number of daughter slugs present in both gaps is very large [see Fig. 7(c)-(d)]. We therefore assume that the temporal fluctuations of the pressure drops resulting from the entrance of exit of a slug in the gaps are very small compared to the mean values of the pressure drops and can be neglected. The validity of this mean-field approximation will be discussed in details in section IV C.

Within this mean-field approach, the total flow rates in each gap are constant over time. Consequently, all twofluid interfaces present in the $i$-th gap $(i=1$ or 2$)$ move at the same constant velocity $v_{i}$. The size of a daughter slug flowing through the gap $(i)$ and the distance between slugs are respectively $L_{d}^{i}=v_{i} t_{f}$ and $\lambda_{i}=v_{i} \tau$ as defined in the previous section. When $\lambda \ll L_{i}$, an estimate of the pressure drop in the gap $(i)$ is $\Delta p \simeq f_{i}\left[\eta_{c} L_{i}+n_{i} L_{d}^{i}\left(\eta_{s}-\right.\right.$ $\left.\left.\eta_{c}\right)\right] \frac{v_{i}}{h^{2}}$ with $n_{i} \simeq L_{i} / \lambda_{i}$, the number of slugs present in this gap and $f_{i}=f\left(\frac{w_{i}}{h}\right)$. The volume conservation of the "mother" slug reads $w_{1} L_{d}^{1}+w_{2} L_{d}^{2}=w L_{d}^{\text {eff }}$. Using this equation, the definitions $L_{d}^{i}$ and $\lambda_{i}$, and the equality of the pressure drops over the two gaps, one easily finds:

$$
\frac{L_{d}^{1}}{L_{d}^{e f f}}=\frac{\lambda_{1}}{\lambda} \simeq \frac{w F \Lambda}{w_{1}(1+F \Lambda)},
$$

and

$$
\frac{L_{d}^{2}}{L_{d}^{e f f}}=\frac{\lambda_{2}}{\lambda} \simeq \frac{w}{w_{2}(1+F \Lambda)}
$$

Since the volume and number of the daughter slugs flowing through the gap $(i)$ are respectively $\Omega_{i} \simeq h w_{i} L_{d}^{i}$ and $n_{i} \simeq L_{i} / \lambda_{i}$, using $\Omega \simeq h w L_{d}^{\text {eff }}$, one predicts:

$$
\begin{gathered}
\phi_{1} \simeq \frac{F \Lambda}{1+F \Lambda}, \\
\phi_{2} \simeq \frac{1}{1+F \Lambda}, \\
n_{1} \simeq \frac{L_{1}}{\lambda} \frac{w_{1}(1+F \Lambda)}{w F \Lambda},
\end{gathered}
$$

and

$$
n_{2} \simeq \frac{L_{2}}{\lambda} \frac{w_{2}(1+F \Lambda)}{w} .
$$


Consequently, this simple model established within the limit $\lambda \ll L_{i}$ predicts that the volume fraction and number of daughter slugs solely depend on the parameters characterizing the asymmetry of an obstacle or a loop. In other words, these physical quantities are independent of the physicochemical and hydrodynamic parameters of the problem. Our experiments presented in section IV A concur well with these results. More quantitatively, when $\Lambda=1$ and $F=2.5$, the predicted volume fraction of the daughter slug in the narrow gap is $\phi_{2} \simeq 0.28$ which correlates with experiments (Fig. 8 and Fig. 9). These results drastically differ from the breakup dynamics against short rectangular obstacles and underlines the importance of the condition $\lambda \ll L_{i}$ for geometrymediated breakups. Within this limit, it is interesting to note that the volume ratio $\frac{\Omega_{2}}{\Omega_{1}}$ of two daughter slugs reads $\frac{1}{F \Lambda}$. For asymmetric loops, it corresponds to the ratio of the hydrodynamic resistances of the two arms only filled with continuous phase. Since both arms are filled with a large number of slugs, this result $\left(\frac{\Omega_{2}}{\Omega_{1}} \propto \frac{1}{\Lambda}\right)$ may seem counterintuitive, although it was inferred based on experimental results in [13]. Our simple model provides theoretical grounds for this intriguing result.

To validate further our theoretical predictions, we work with asymmetric loops and we compare theoretical predictions for the number and volume fractions of the produced daughter for both geometries.

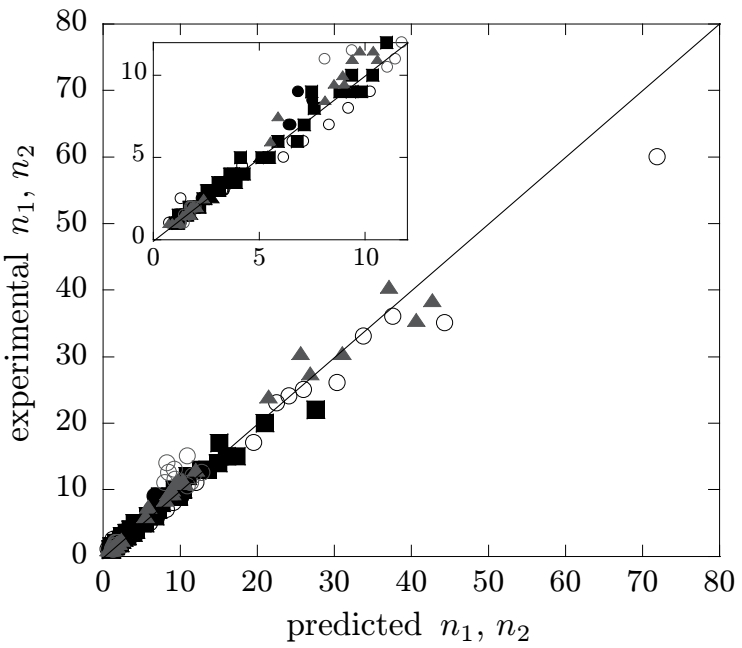

FIG. 11. Comparison between the experimental and predicted average numbers of slugs $n_{i}$ obtained the gap $(i)$ after breakup. Inset: magnification at small number of slugs. The shapes of the symbols correspond to different liquidliquid systems and values of $\eta_{d}$ : water-glucose mixtures in hexadecane with (०) $\eta_{d}=1 \mathrm{mPa} \mathrm{s}$, (ם) $\eta_{d}=2.7 \mathrm{mPa} \mathrm{s}$, and (ム) $\eta_{d}=7.2 \mathrm{mPa} \mathrm{s}$, and water in a viscous silicone oil $(\bullet)$ $\eta_{d}=1 \mathrm{mPa}$ s. Colors stands for experiments either carried out using long obstacles (black) or asymmetric loops (grey).

As shown in Fig. 11, the model well describes the number of daughter slugs present in both gaps of an obstacle or in the arms of a loop. Also, when comparing exper- imental and predicted volume fractions $\phi_{1}$ and $\phi_{2}$, the mean-field model well captures experiments (Fig. 12).

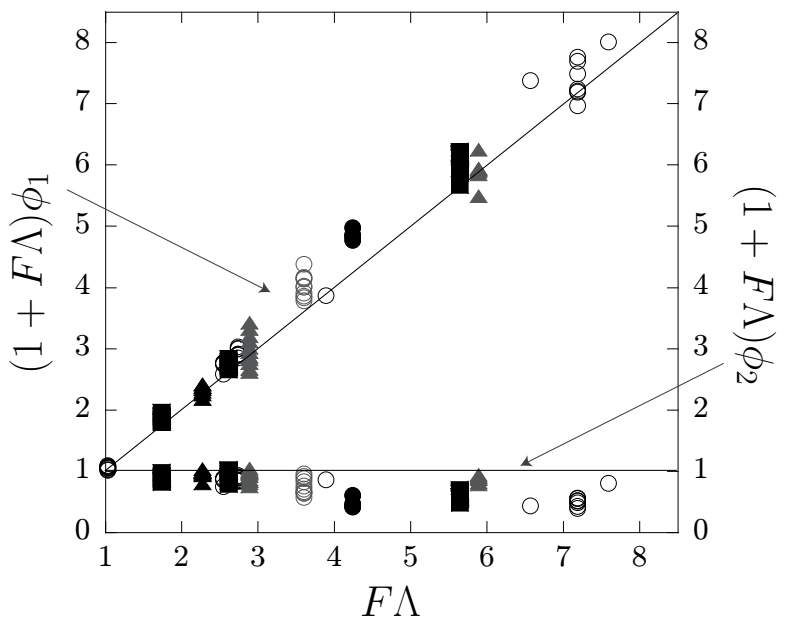

FIG. 12. Comparison between experiments and theoretical predictions of the volume fraction $\phi_{1}$ (left y-axis) and $\phi_{2}$ (right y-axis) of the slugs produced after breakup. The shape of the symbols and their colors are identical to those of Fig. 11.

\section{Validity of the mean-field approach}

We now discuss the conditions for the validity of our mean-field approach. Our first assumption requires that $n_{i} \gg 1$ with $i=1$ or 2 . Using eqn (9) and eqn (10), this condition can be expressed as:

$$
\lambda \ll \min \left(\lambda_{c}^{(1)}, \lambda_{c}^{(2)}\right)
$$

with $\lambda_{c}^{(1)}=L_{1} \frac{w_{1}(1+F \Lambda)}{w F \Lambda}$ and $\lambda_{c}^{(2)}=L_{2} \frac{w_{2}(1+F \Lambda)}{w}$.

The mean-field approximation also requires the temporal fluctuations of the pressure drop $\Delta p(t)$ caused by the entrance, exit, and motion of the slugs in both gaps to be negligible compared to its mean value. Following the description of the motion of slugs in channels having a constant cross-section given in section IIIB 1, the pressure drop can be written $\Delta p(t)=\frac{f_{i} v_{i}}{h^{2}}\left[\eta_{c} L_{i}+\left(\eta_{s}-\eta_{c}\right) L_{i}^{s}(t)\right]+\epsilon_{i}(t) \frac{2 \gamma}{w_{i}}\left(1+\frac{w_{i}}{h}\right)$. In this expression, $L_{i}^{s}(t)$ is the total length occupied at $t$ by the slugs in the gap $(i)$ and $\epsilon_{i}(t)$ can take three possible values, 1,0 , or -1 depending on whether at $t$, the difference between the number of front and rear edges of slugs present in this gap is 1,0 , or -1 . Since $L_{i}$ is generally not commensurate with $\lambda_{i}$, one can write $L_{i}^{s}(t)=N_{i} L_{d}^{i}+\ell(t)$ where $N_{i}=$ floor $\left(\frac{L_{i}}{\lambda_{i}}\right)$ and $\ell(t)$ is a length smaller than $L_{d}^{i}$. $\quad N_{i}$ represents the minimum number of slugs that can entirely fit in the gap $(i)$ and $\ell(t)$ corresponds to the excess length due to the partial entrance and exit of slugs through this gap at time $t$. When $\lambda_{i} \ll L_{i}, N_{i} \simeq \frac{L_{i}}{\lambda_{i}}$, and using eqn (5) and eqn (6), the pressure drop can be written $\Delta p(t) \simeq$ 
$\frac{f_{i} v_{i} \eta_{c} L_{i}}{h^{2}}\left[\left(1+\Delta \eta \frac{L_{d}^{e f f}}{\lambda}\right)+\Delta \eta \frac{\ell(t)}{L_{i}}+\epsilon_{i}(t) \frac{2 v}{\mathcal{C} v_{i}} Z_{i}\left(1+\frac{w_{i}}{h}\right)\right]$ with $Z_{i}=\left(f_{i} h^{-2} w_{i} L_{i}\right)^{-1}$.

Neglecting the temporal fluctuations of the pressure drop compared to its mean value therefore requires the two following conditions to be satisfied for $i=1$ and 2 :

(a) $|\Delta \eta| \frac{L_{d}^{i}}{L_{i}} \ll 1+\Delta \eta \frac{L_{d}^{e f f}}{\lambda}$,

(b) $\frac{2 v}{\mathcal{C} v_{i}} Z_{i}\left(1+\frac{w_{i}}{h}\right) \ll 1+\Delta \eta \frac{L_{d}^{e f f}}{\lambda}$.

The condition (a) is satisfied when the resistive length added by one slug to the gap $(i)$ is negligible compared to the effective resistive length of this gap. The condition (b) is fulfilled when the pressure drop accounting for curved interfaces in the gap $(i)$ can be neglected compared to the pressure drop resulting from the flow of slugs and continuous phase over this gap. We will next show that these two conditions, which depend on whether $\Delta \eta \geq 0$ or $\Delta \eta<0$, imply restrictions on $\lambda$ and $\mathcal{C}$.

For $\Delta \eta \geq 0$, the condition (a) is fulfilled whenever eqn (11) is satisfied. Using eqn (5), eqn (6), and $\frac{v}{v_{i}}=\frac{\lambda}{\lambda_{i}}$, one easily finds that the condition (b) reads:

$$
\mathcal{C} \gg \min \left(\mathcal{C}_{c}^{+}, \frac{1+\frac{w_{2}}{h}}{1+\frac{w_{1}}{h}} \mathcal{C}_{c}^{+}\right)
$$

with $\mathcal{C}_{c}^{+}=2 \frac{w_{1}}{w} Z_{1}\left(1+\frac{w_{1}}{h}\right) \frac{1+F \Lambda}{F \Lambda} \frac{1}{1+\Delta \eta \frac{L_{d}^{e f f}}{\lambda}}$.

It is interesting to note that the expression of $\mathcal{C}_{c}^{+}$can be simplified within the two following limits:

$$
\begin{aligned}
& \text { - } \lambda \gg \Delta \eta L_{d}^{e f f}, \mathcal{C}_{c}^{+} \simeq 2 \frac{w_{1}}{w} Z_{1}\left(1+\frac{w_{1}}{h}\right) \frac{1+F \Lambda}{F \Lambda} \\
& \text { - } \lambda \ll \Delta \eta L_{d}^{e f f}, \mathcal{C}_{c}^{+} \simeq 2 \frac{w_{1}}{w} Z_{1}\left(1+\frac{w_{1}}{h}\right) \frac{1+F \Lambda}{F \Lambda} \frac{\lambda}{\Delta \eta L_{d}^{e f f}} .
\end{aligned}
$$

To summarize, when $\Delta \eta \geq 0$, the mean-field approximation is valid whenever $\lambda$ and $\mathcal{C}$ satisfy both eqn (11) and eqn (12). Note that when $\Delta \eta=0$, only eqn (12) has to be fulfilled.

For $\Delta \eta<0$, the condition $(a)$ becomes $-\Delta \eta \frac{L_{d}^{i}}{L_{i}} \ll$ $1+\Delta \eta \frac{L_{d}^{e f f}}{\lambda}$. Using eqn (5) and eqn (6), and writing $\frac{L_{d}^{i}}{L_{i}}$ as $\frac{L_{d}^{i}}{L_{d}^{\text {eff }}} \frac{L_{d}^{\text {eff }}}{L_{i}}$, one shows that $\frac{L_{d}^{i}}{L_{i}}=\frac{L_{d}^{\text {eff }}}{\lambda_{c}^{(i)}}$. Since $\lambda \ll \lambda_{c}^{(i)}$ [see eqn (11)], the condition (a) is fulfilled only when

$$
\lambda \gg-\Delta \eta L_{d}^{e f f} .
$$

Using eqn (13), it is straightforward to show that the condition (b) can be written as

$$
\mathcal{C} \gg \min \left(\mathcal{C}_{c}^{-}, \frac{1+\frac{w_{2}}{h}}{1+\frac{w_{1}}{h}} \mathcal{C}_{c}^{-}\right)
$$

with $\mathcal{C}_{c}^{-}=2 \frac{w_{1}}{w} Z_{1}\left(1+\frac{w_{1}}{h}\right) \frac{1+F \Lambda}{F \Lambda}$.
To summarize the case $\Delta \eta<0$, the validity of our mean-field approximation requires that $\lambda$ and $\mathcal{C}$ satisfy eqn (11), eqn (13), and eqn (14).

Note that for the the long rectangular obstacles $(F>1$ and $\Lambda=1)$ and loops $(F=1$ and $\Lambda>1)$ studied here, the conditions for the capillary number given in eqn (12) and eqn (14) respectively read:

$$
\mathcal{C} \gg 2 \frac{w_{1}}{w} Z_{1}\left(1+\frac{w_{2}}{h}\right) \frac{1+F \Lambda}{F \Lambda} \frac{1}{1+\Delta \eta \frac{L_{d}^{e f f}}{\lambda}}
$$

and

$$
\mathcal{C} \gg 2 \frac{w_{1}}{w} Z_{1}\left(1+\frac{w_{2}}{h}\right) \frac{1+F \Lambda}{F \Lambda} .
$$

\section{CONCLUSION}

Investigating the fragmentation of isolated slugs (large drops and bubbles) against rectangular obstacles, we have shown the existence of a critical capillary number needed to break a slug; this result concurs with a previous study [23]. Above this critical capillary number, a slug breaks into two daughter slugs that flow through both sides of the obstacle. A thorough study of their respective volumes has shown a complex dependance on the numerous parameters of the problem, the volume and velocity of the initial slug, the viscosity contrast between dispersed and continuous phases, the surface tension, and the geometrical features of the obstacle. We show that a theoretical framework initially introduced in [23] well describes experimental findings.

Working within the limit of very long obstacles compared to the distance between slugs allows for slug-toslug interactions. We show that such interactions drastically change the flow behavior and the breakup dynamics. Indeed for this flow configuration, the ratio of the volume of the daughter slugs over the volume of the initial slug is independent of the hydrodynamic parameters and physicochemical properties and solely depends on the geometrical features of the device. Inspired by recent works on droplet traffic at microfluidic junctions [35, 36], we introduce a mean-field approximation and build on the modeling of the breakup dynamics for isolated slugs to rationalize experiments in the presence of slug-to-slug interactions. The resulting model well describes both qualitatively and quantitatively our experimental findings. The predictions of this model for the ratio of the volumes of the two daughter slugs also provide theoretical grounds for an unexplained result reported in the pioneering work on geometry-mediated breakups in microfluidic devices [13]. The conditions of validity of the mean-field approach, i.e the range of applied capillary numbers and distance between slugs, are also discussed. These results provide an insightful description of geometrically-mediated breakups of drops and bubbles in confined environments and could be useful for engineering bidisperse emulsions and foams [8]. 
[1] M. Joanicot and A. Ajdari, Science, 2005, 309, 887-888.

[2] R. B. Fair, Microfluid. Nanofluid., 2007, 3, 245-281.

[3] S.-Y. Teh, R. Lin, L.-H. Hung and A. P. Lee, Lab Chip, 2008, 8, 198-220.

[4] N. Lorber, F. Sarrazin, P. Guillot, P. Panizza, A. Colin, B. Pavageau, C. Hany, P. Maestro, S. Marre, T. Delclos, C. Aymonier, P. Subra, L. Prat, C. Gourdon and E. Mignard, Lab Chip, 2011, 11, 779-787.

[5] W. Engl, R. Backov and P. Panizza, Curr. Opin. Colloid Interface Sci., 2008, 13, 206-216.

[6] S. Okushima, T. Nisisako, T. Torii and T. Higushi, Langmuir, 2004, 20, 9905-9908.

[7] L. Y. Chu, A. S. Utada, R. K. Shah, J. -W. Kim and D. A. Weitz, Angew. Chem. Int. Ed., 2007, 46, 8970-8974.

[8] P. Panizza, W. Engl, C. Hany and R. Backov, Colloids Surf. A, 2008, 312, 24-31.

[9] R. F. Shepherd, J. C. Conrad, S. K. Rhodes, D. R. Link, M. Marquez, D. A. Weitz and J. A. Lewis, Langmuir, 2006, 22, 8618-8622.

[10] Z. Nie, W. Li, M. Seo, S. Xu and E. Kumacheva, J. Am. Chem. Soc., 2006, 128, 9408-9412.

[11] Y. Hennequin, N. Pannacci, C. P. de Torres, G. TetradisMeris, S. Chapuliot, E. Bouchaud and P. Tabeling, Langmuir, 2009, 25, 7857-7861.

[12] J. W. Kim, A. S. Utada, A. Fernández-Nieves, Z. Hu and D. A. Weitz, Angew. Chem. Int. Ed., 2007, 46, 18191822 .

[13] D. R. Link, S. L. Anna, D. A. Weitz and H. A. Stone, Phys. Rev. Lett., 2004, 92, 054503.

[14] M. De Menech, Phys. Rev. E: Stat., Nonlinear, Soft Matter Phys., 2006, 73, 031505.

[15] A. M. Leshansky and L. M. Pismen, Phys. Fluids 21, 023303 (2009)

[16] A. M. Leshansky, S. Afkhami, M.-C. Jullien and P. Tabeling, Phys. Rev. Lett., 2012, 108, 264502.

[17] M.-C. Jullien, M.-J. Tsng Mui Ching, C. Cohen, L. Menetrier and P. Tabeling, Phys. Fluids, 2009, 21, 072001.

[18] T. Fu, Y. Ma D. Funfschilling, and H. Z. Li, Chem. Eng. Sci., 2011, 66, 4184-4195.

[19] Y. Wu, T. Fu, C. Zhu, Y. Lu, Y. Ma and H. Z. Li, Microfluid. Nanofluid., 2012, 13, 723-733.

[20] L. Ménétrier-Deremble and P. Tabeling, Phys. Rev. E: Stat., Nonlinear, Soft Matter Phys., 2006, 74, 035303(R).

[21] M. Yamada, S. Doi, H. Maenaka, M. Yasuda and M. Seki, J. Colloid Interface Sci., 2008, 321, 401-407.

[22] S. Protière, M. Z. Bazant, D. A. Weitz and H. A. Stone, EPL, 2010, 92, 54002.

[23] L. Salkin, L. Courbin and P. Panizza, Phys. Rev. E: Stat., Nonlinear, Soft Matter Phys., 2012, 86, 036317.

[24] D. R. Link, E. Grasland-Mongrain, A. Duri, F. Sarrazin, Z. Cheng, G. Cristobal, M. Marquez and D. A. Weitz, Angew. Chem. Int. Ed., 2006, 45, 2556-2560.
[25] T. Cubaud, Phys. Rev. E: Stat., Nonlinear, Soft Matter Phys., 2009, 80, 026307.

[26] M. T. Guo, A. Rotem, J. A. Heyman and D. A. Weitz, Lab Chip, 2012, 12, 2146-2155.

[27] A. C. Hatch, J. S Fisher, A. R. Tovar, A. T. Hsieh, R. Lin, S. L. Pentoney, D. L. Yang and A. P. Lee, Lab Chip, 2011, 11, 3838-3845.

[28] J. Clausell-Tormos, A. D Griffiths and C. A. Merten, Lab Chip, 2010, 10, 1302-1307.

[29] A. R. Abate and D. A. Weitz, Lab Chip, 2011, 11, 19111915.

[30] F. Jousse, R. Farr, D. R. Link, M. J. Fuerstman and P. Garstecki, Phys. Rev. E: Stat., Nonlinear, Soft Matter Phys., 2006, 74, 036311.

[31] M. Schindler and A. Ajdari, Phys. Rev. Lett., 2008, 100, 044501.

[32] M. Belloul, W. Engl, A. Colin, P. Panizza and A. Ajdari, Phys. Rev. Lett., 2009, 102, 194502.

[33] O. Cybulski and P. Garstecki, Lab Chip, 2010, 10, 484493.

[34] R. Jeanneret, J. P. Vest, and D. Bartolo, Phys. Rev. Lett., 2012, 108, 034501.

[35] D. A. Sessoms, A. Amon, L. Courbin and P. Panizza, Phys. Rev. Lett., 2010, 105, 154501.

[36] T. Glawdel C. Elbuken and C. L. Ren, Lab Chip, 2011, 11, 3774-3784.

[37] J. C. McDonald, D. C. Duffy, J. R. Anderson, D. T. Chiu, H. Wu, O .J. A. Schueller and G. M. Whitesides, Electrophoresis, 2000, 21, 27-40.

[38] S. L. Anna, N. Bontoux and H. A. Stone, Appl. Phys. Lett., 2003, 82, 364-366.

[39] H. Bruus, Theoretical Microfluidics, Oxford University Press, 2008.

[40] Note that if $\ell$ is not commensurate with $\lambda$, the contributions of the rear and front edges usually do not cancel out. Their contribution to the pressure drop can however be neglected as soon as the capillary number becomes sufficiently large. See section IV C for a detailed discussion of this point.

[41] D. A. Sessoms, M. Belloul, W. Engl, M. Roche, L. Courbin and P. Panizza, Phys. Rev. E: Stat., Nonlinear, Soft Matter Phys., 2009, 80, 016317.

[42] V. Labrot, M. Schindler, P. Guillot, A. Colin and M. Joanicot, Biomicrofluidics, 2009, 3, 012804.

[43] S. A. Vanapalli, A. G. Banpurkar, D. van den Ende, M. H. G. Duits and F. Mugele, Lab Chip, 2009, 9, 982990.

[44] H. A. Stone, Annu. Rev. Fluid Mech., 1994, 26, 65-102.

[45] A. Vananroye, P. V. Puyvelde and P. Moldenaers, Langmuir, 2006, 22, 3972-3974.

[46] A. Schmit, L. Salkin, L. Courbin and P. Panizza (unpublished). 